\title{
Measurement of the absorption coefficient of sound absorbing materials under a synthesized diffuse acoustic field
}

\author{
Olivier Robin, ${ }^{\text {a) }}$ Alain Berry, ${ }^{\text {a) }}$ Olivier Doutres, and Noureddine Atalla \\ Groupe d'Acoustique de l'Université de Sherbrooke, Université de Sherbrooke, Sherbrooke, \\ Quebec J1K2R1, Canada \\ olivier.robin@usherbrooke.ca,alain.berry@usherbrooke.ca, \\ olivier.doutres@usherbrooke.ca,noureddine.atalla@usherbrooke.ca
}

\begin{abstract}
This letter proposes an experimental method to estimate the absorption coefficient of sound absorbing materials under a synthesized diffuse acoustic field in free-field conditions. Comparisons are made between experiments conducted with this approach, the standard reverberant room method, and numerical simulations using the transfer matrix method. With a simple experimental setup and smaller samples than those required by standards, the results obtained with the proposed approach do not exhibit non-physical trends of the reverberant room method and provide absorption coefficients in good agreement with those obtained by simulations for a laterally infinite material.
\end{abstract}

(C) 2014 Acoustical Society of America

PACS numbers: 43.55.Ev, 43.38.Md [NX]

Date Received: March 1, $2014 \quad$ Date Accepted: May 21, 2014

\section{Introduction}

The absorption coefficient of sound absorbing materials is classically measured using the reverberant room method ${ }^{1,2}$ and the impedance tube method. ${ }^{3,4}$ The impedance tube method allows measuring the normal incidence sound absorption on small samples with mounting conditions that can have nonnegligible influence on measured results. ${ }^{5}$ The reverberant room method gives access to the absorption coefficient under a Diffuse Acoustic Field (DAF) excitation, which is more representative of practical utilization of sound absorbing materials. Testing large samples as recommended by standards may nevertheless prevent the obtention of a logarithmic sound decay rate in the reverberant room, but measurements on small material samples lead to larger absorption results than predicted by theory as well. ${ }^{6,7}$ Significant deviations on measured absorption coefficient were also observed in round robin tests ${ }^{7,8}$ depending on test room volume, sample mounting, and diffusers effect.

Apart from the Alpha Cabin small reverberant room technique, ${ }^{9}$ several studies have proposed approaches for estimating the absorption coefficient under a DAF excitation while removing both constraints of sample size and room effects. Takahashi et al. ${ }^{10}$ suggested a measurement using two microphones located close to a material and diffuse ambient noise in a room. This concept was investigated using a pressure-velocity probe and loudspeakers as sound sources in different rooms for measuring ensemble averaged surface normal impedance. ${ }^{11}$ Using boundary element method simulations results, ensemble averaging of contributions of spatially distributed sources on a hemisphere surrounding a material was used to simulate random incidence effects by Otsuru et al. ${ }^{12}$ and was shown to be effective for reducing edge effects. Finally, Kuang et al. ${ }^{13}$ suggested the use of a highly directional parametric

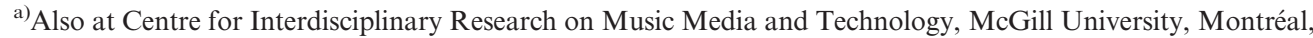
Quebec H3A 1E3, Canada.
} 
loudspeaker to measure the absorption coefficient of a material under various incidence angles corresponding to different positions of the parametric loudspeaker on a circular arc. A diffuse-field absorption coefficient was derived by equally averaging measurements over incidence angles (Takahashi ${ }^{10}$ followed a similar idea using a classical loudspeaker).

This letter proposes a method for the estimation in free-field conditions of the absorption coefficient of a sound absorbing material under a synthesized DAF excitation at the material surface by using a synthetic array of acoustic monopoles facing the material. The reproduction of random pressure fields on plane surfaces was originally developed for the vibroacoustic testing of plane panels under DAF or turbulent boundary layer excitations. ${ }^{14-16}$ In the present case, a database of measured reflection coefficients at normal and oblique incidence angles is first generated with the classical two-microphone approach and a source-image model, ${ }^{17}$ using a point source that is moved over a plane parallel to the material surface. An approach based on Planar Nearfield Acoustic Holography (P-NAH) ${ }^{15}$ is then used to calculate a CrossSpectral Density (CSD) matrix of source amplitudes to reproduce a target DAF on the material's surface. Coupling this calculated matrix to the measured reflection coefficients database, in a post processing phase, allows estimating the absorption coefficient under a synthetic DAF. Note that another approach based on wave field synthesis ${ }^{14}$ could also be used for the calculations of the CSD matrix of source amplitudes.

\section{Description of the proposed method}

\subsection{Absorption coefficient under a point source}

As a preamble to the measurement of the reflection coefficient under a synthesized excitation using an array of point sources, Fig. 1(a) describes a simpler situation. A single point source is positioned at a given position $i$ at a height $z=z_{s}$ above a layer of porous material. Two microphones denoted $M 1$ and $M 2$ are placed above the porous material and centered on its surface at heights $z=z_{1}$ and $z=z_{2}$, respectively. Under the assumption of an ideal point source [defined by its volume acceleration $\dot{q}_{i}(\omega)$ ], the acoustic field at any of the microphone positions is a superposition of two spherical acoustic waves, generated by the source $\dot{q}_{i}(\omega)$ and the corresponding image source $\dot{q}_{i}^{\prime}(\omega)$ positioned at a distance $r_{i}$ and a distance $r_{i}^{\prime}$ from the receiver, respectively. For a small separation of the two microphones so that the angle $\theta_{i}$ is almost identical for both microphones, the measured acoustic pressure $\tilde{p}_{i j}\left(\theta_{i}, \omega\right)$ for a given position $i$ of the point source at microphone $M_{j}(j=1,2)$ can be written
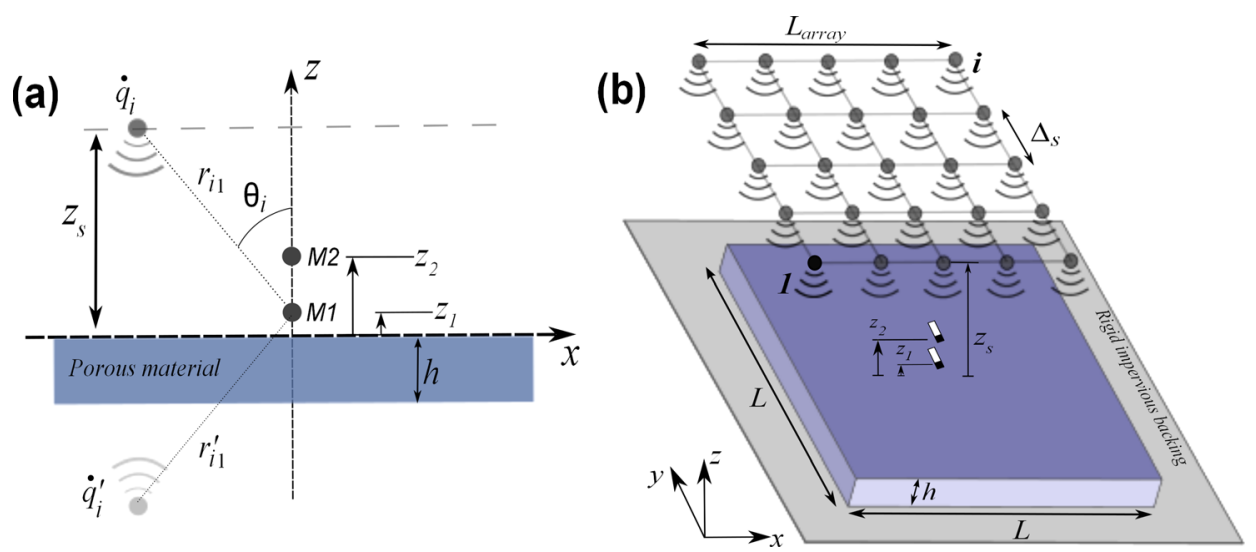

Fig. 1. (Color online) (a) Description of the problem and coordinate system for a spherical wave model using a single point source. (b) Description of the problem using a $i$-source array (the sidelength of the virtual array is $L_{\text {array }}$, with uniformly distributed sources positioned with the same source separation $\Delta_{s}$ ). 


$$
\tilde{p}_{i j}\left(\theta_{i}, \omega\right)=\rho_{0} \dot{q}_{i}(\omega) \frac{e^{-j k_{0} r_{i j}}}{r_{i j}}+R\left(\theta_{i}, \omega\right) \rho_{0} \dot{q}_{i}(\omega) \frac{e^{-j k_{0} r_{i j}^{\prime}}}{r_{i j}^{\prime}},
$$

with $\rho_{0}$ the air mass density, $\omega$ the angular frequency, $k_{0}$ the acoustic wavenumber ( $k_{0}=\omega / c_{0}$ with $c_{0}$ the speed of sound), $r_{i j}$ the distance between the source at the $i$ th position and the microphone $M_{j}, r_{i j}^{\prime}$ the distance between the image source and the microphone $M_{j}$ and $R\left(\theta_{i}, \omega\right)$ is the reflection coefficient of the material surface corresponding to the $i$ th position of the point source. The measurement of either $\tilde{p}_{i j}\left(\theta_{i}, \omega\right) / \dot{q}_{i}(\omega)$ at each microphone or $H\left(\theta_{i}, \omega\right)\left(=\tilde{p}_{i 2}\left(\theta_{i}, \omega\right) / \tilde{p}_{i 1}\left(\theta_{i}, \omega\right)\right)$ allows calculating the reflection coefficient for a given incidence angle using the classical relation ${ }^{17}$

$$
R\left(\theta_{i}, \omega\right)=\frac{\frac{e^{-j k_{0} r_{i 2}}}{r_{i 2}}-H\left(\theta_{i}, \omega\right) \frac{e^{-j k_{0} r_{i 1}}}{r_{i 1}}}{H\left(\theta_{i}, \omega\right) \frac{e^{-j k_{0} r_{i 1}^{\prime}}}{r_{i 1}^{\prime}}-\frac{e^{-j k_{0} r_{i 2}^{\prime}}}{r_{i 2}^{\prime}}} .
$$

The corresponding absorption coefficient is then deduced using the relation $\alpha\left(\theta_{i}, \omega\right)=1-\left|R\left(\theta_{i}, \omega\right)\right|^{2}$.

\subsection{Reflection coefficient under a synthesized acoustic field}

Figure 1(b) illustrates the proposed approach. A square sample of porous material of side length $L$ and thickness $h$ is placed on a rigid impervious backing. The two microphones $M 1$ and $M 2$ and the array center point source are centered on the material surface. Using the two-microphone method described in the previous section, the reflection coefficient can be measured under various incidence angles corresponding to successive source positions $i$ of point sources, thus creating a virtual array of monopoles in front of the material surface. The Green's functions corresponding to the propagation from the real (respectively, image) point source to the microphone $M_{j}$ will now be denoted $g_{\mathrm{ij}}(\omega)=e^{-j k_{0} r_{i j}} / r_{i j}$ (respectively, $g_{i j}^{\prime}(\omega)=e^{-j k_{0} r_{i j}^{\prime}} / r_{i j}^{\prime}$ ). The sound pressure measured at the microphone $M 1$ due to the contribution of all point sources is a simple summation

$$
\tilde{p}_{1}(\omega)=\sum_{i} \dot{q}_{i}(\omega) \rho_{0} g_{i 1}(\omega)+\sum_{i} R\left(\theta_{i}, \omega\right) \dot{q}_{i}(\omega) \rho_{0} g_{i 1}^{\prime}(\omega)=\dot{\mathbf{Q}}^{T} \mathbf{g}_{1}+\dot{\mathbf{Q}}^{T} \mathbf{h}_{1},
$$

where $\dot{\mathbf{Q}}=\left\{\cdots \dot{q}_{i}(\omega) \cdots\right\}^{T}, \mathbf{g}_{\mathbf{1}}=\left\{\cdots \rho_{0} g_{i 1}(\omega) \cdots\right\}^{T}, \mathbf{h}_{\mathbf{1}}=\left\{\cdots R\left(\theta_{i}, \omega\right) \rho_{0} g_{i 1}^{\prime}(\omega) \cdots\right\}^{T}$, and ${ }^{T}$ denotes the non-conjugate transpose. Introducing $R_{\text {synth }}(\omega)$, the reflection coefficient of the material surface under a synthesized pressure field, the sound pressure measured at the microphone $M 1$ can also be written

$$
\tilde{p}_{1}(\omega)=\sum_{i} \dot{q}_{i}(\omega) \rho_{0} g_{i 1}(\omega)+R_{\text {synth }}(\omega) \sum_{i} \dot{q}_{i}(\omega) \rho_{0} g_{i 1}^{\prime}(\omega)=\dot{\mathbf{Q}}^{T} \mathbf{g}_{1}+R_{\text {synth }}(\omega) \dot{\mathbf{Q}}^{T} \mathbf{g}_{1}^{\prime},
$$

where $\mathbf{g}_{1}^{\prime}=\left\{\cdots \rho_{0} g_{i 1}^{\prime}(\omega) \cdots\right\}^{T}, \dot{\mathbf{Q}}^{T} \mathbf{g}_{\mathbf{1}}$ is the synthetic incident pressure field at microphone $M 1$, and $\dot{\mathbf{Q}}^{T} \mathbf{g}_{1}^{\prime}$ is the synthetic pressure field that would be reflected by material surface if it was rigid, at the same microphone. Comparing Eqs. (3) and (4) leads to

$$
\dot{\mathbf{Q}}^{T} \mathbf{h}_{\mathbf{1}}=R_{\text {synth }}(\omega) \dot{\mathbf{Q}}^{T} \mathbf{g}_{\mathbf{1}}^{\prime},
$$

and Eqs. (3)-(5) also stand for the second microphone $M 2$. Note that both microphones are needed for the calculation of the individual reflection coefficients $R\left(\theta_{i}, \omega\right)$, but the calculation of $R_{\text {synth }}(\omega)$ requires only one of them. 
To relate the synthesized reflection coefficients to the CSD matrix of source volume accelerations $\mathbf{S}_{\mathbf{Q} \mathbf{Q}}\left(=\dot{\mathbf{Q}} * \dot{\mathbf{Q}}^{T}\right)$, Eq. (5) is multiplied by its conjugate transpose (noted ${ }^{H}$ ) which gives

$$
\mathbf{h}_{\mathbf{1}}{ }^{H} \mathbf{S}_{\mathbf{Q} \mathbf{Q}} \mathbf{h}_{\mathbf{1}}=\left|R_{\text {synth }}(\omega)\right|^{2} \mathbf{g}_{1}^{\prime}{ }^{H} \mathbf{S}_{\mathbf{Q} \mathbf{Q}} \mathbf{g}_{1}^{\prime},
$$

and the squared reflection coefficient therefore takes the form

$$
\left|R_{\mathrm{synth}}(\omega)\right|^{2}=\frac{\mathbf{h}_{\mathbf{1}}{ }^{H} \mathbf{S}_{\mathbf{Q} \mathbf{Q}} \mathbf{h}_{\mathbf{1}}}{\mathbf{g}_{\mathbf{1}}^{\prime}{ }^{H} \mathbf{S}_{\mathbf{Q} \mathbf{Q}} \mathbf{g}_{\mathbf{1}}^{\prime}} .
$$

With a database of measured reflection coefficients $R\left(\theta_{i}, \omega\right)$ and a calculated CSD matrix of source volume accelerations $\mathbf{S}_{\mathbf{Q} \dot{Q}}$, Eq. (7) provides the squared reflection coefficient $\left|R_{\text {synth }}(\omega)\right|^{2}$ under a synthesized pressure field at a post-processing phase. The corresponding absorption coefficient can be deduced using the relation $\alpha_{\text {synth }}(\omega)=1-\left|R_{\text {synth }}(\omega)\right|^{2}$.

The CSD matrix of source volume acceleration can be calculated using either the wave field synthesis approach ${ }^{14}$ or the P-NAH approach ${ }^{15}$ (in this letter, only P$\mathrm{NAH}$ is used), with a target pressure field defined by the CSD of a DAF. ${ }^{18}$

\section{Description of measurements and simulations}

Melamine foam samples of two different thicknesses and areas were tested. Their properties were measured in the Acoustic Materials Characterization Labs of Universite de Sherbrooke, using the methods described by Doutres et al. ${ }^{19}$ and given in Table 1 .

Reverberant room absorption tests of melamine foam of $0.0762 \mathrm{~m} \mathrm{(3} \mathrm{in.)} \mathrm{and}$ $0.0508 \mathrm{~m}$ ( 2 in.) thicknesses were made in the National Research Canada (NRC) reverberant room (volume $\approx 258 \mathrm{~m}^{3}$ ) and in the Groupe d'Acoustique de l'Université de Sherbrooke (GAUS) reverberant room (volume $\approx 143 \mathrm{~m}^{3}$ ), respectively. The specimen area was $5.94 \mathrm{~m}^{2}(2.438 \mathrm{~m} \times 2.438 \mathrm{~m})$ for the 3 inches melamine foam and $3.35 \mathrm{~m}^{2}$ $\left(1.829 \times 1.829 \mathrm{~m}^{2}\right)$ for the 2 inches melamine foam, so that only the first case fulfills the ASTM C423 requirements in terms of sample area. For both experiments, samples were laid directly against the room floor, and their perimeters were sealed by wood framing [see Fig. 2(a) for tests at NRC]. Sabine absorption coefficients were calculated following standards procedures. ${ }^{1,2}$

Figures 2(b) and 2(c) illustrate the experiments conducted in an anechoic room using the proposed approach. The specimen area was $2.11 \mathrm{~m}^{2}$ $(1.320 \mathrm{~m} \times 1.600 \mathrm{~m})$ for the 3 -in. melamine foam and $1.49 \mathrm{~m}^{2}(1.219 \mathrm{~m} \times 1.219 \mathrm{~m})$ for the 2-in. melamine foam. Each sample was laid on a rigid impervious backing, made up from a $1 / 2$ in. thick medium density fiberboard panel covered with a $1 / 32$ in. steel plate. An omnidirectional point source (LMS Qsource midfrequency volume source) was manually translated using a rigid frame on a mesh of $7 \times 7$ positions above the material surface at a height $z_{s}=0.2 \mathrm{~m}$ with the center source position corresponding to the normal incidence case. The source separation $\Delta_{s}$ was set to $0.15 \mathrm{~m}$ with a corresponding virtual array sidelength $L_{\text {array }}$ of $0.9 \mathrm{~m}$. The maximum incidence angle $\theta_{\max }$

Table 1. Material parameters used in calculations.

\begin{tabular}{lcccccc}
\hline \hline $\begin{array}{c}\text { Parameter } \\
\text { (unit) }\end{array}$ & $\begin{array}{c}\text { Tortuosity } \\
\alpha_{\infty}(-)\end{array}$ & $\begin{array}{c}\text { Porosity } \\
\phi(-)\end{array}$ & $\begin{array}{c}\text { Resistivity } \\
\sigma\left(\mathrm{Nm}^{-4} \mathrm{~s}\right)\end{array}$ & $\begin{array}{c}\text { Viscous } \\
\text { length } \\
\Lambda(\mu \mathrm{m})\end{array}$ & $\begin{array}{c}\text { Thermal } \\
\text { length } \\
\Lambda^{\prime}(\mu \mathrm{m})\end{array}$ & $\begin{array}{c}\text { Foam mass } \\
\text { density } \rho_{1} \\
\left(\mathrm{~kg} \mathrm{~m}^{-3}\right)\end{array}$ \\
\hline Value & 1 & 0.99 & 10900 & 100 & 130 & 8.8 \\
\hline \hline
\end{tabular}



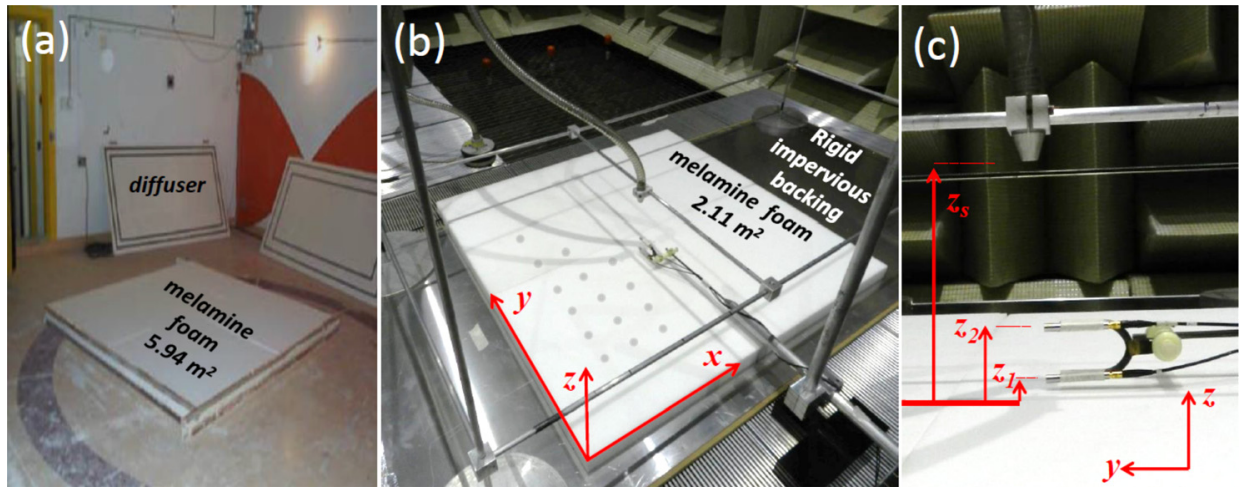

Fig. 2. (Color online) Pictures of measurements performed on the melamine foam of 3 in. thickness: (a) Reverberant room measurement at NRC. (b) Measurement under a synthetic array in an anechoic room (the two microphones and the source are positioned at the center of the sample, and some successive source positions are indicated by gray circles). (c) Close view of the two microphones and source positioned above the material's surface for the normal incidence measurement.

that can be included in the database of measured reflection coefficients is defined by the source to reproduction plane separation $z_{s}$ and the largest reproduction source to microphones distance. In the present case, $\theta_{\max }=\tan ^{-1}\left(L_{\text {array }} / \sqrt{2} z_{s}\right) \approx 72^{\circ}$. The microphones $M 1$ and $M 2$ (BSWA MPA416 1/4 in. microphones) were positioned at the center of the sample at heights $z_{1}=15 \mathrm{~mm}$ and $z_{2}=59 \mathrm{~mm}$, respectively. Amplitude calibration was performed for both microphones before measurements, and the volume acceleration of the point source was derived from an internal sensor. For each source position, a white noise was used to drive the point source and the transfer functions between the sound pressure at both microphones and the source volume acceleration were measured from 170 to $2000 \mathrm{~Hz}$, the low frequency limit being intrinsic to low frequency limitations of the volume source. With time averaging over $30 \mathrm{~s}$ for each successive test, a database corresponding to the 49 sources positions was obtained in approximately half an hour.

Finally, simulations based on the Transfer Matrix Method (TMM) ${ }^{20}$ were performed. The layer of homogeneous material of infinite extent and backed by a hard wall was modeled under a limp frame assumption. ${ }^{20}$ The parameters given in Table 1 were used in simulations to compute the characteristic impedance $Z_{c e q}$ and the wavenumber $k_{e q}$ of the equivalent fluid element via the Johnson-Champoux-Allard model. ${ }^{20}$ Because the DAF theoretically reproduced with the synthetic array implies a maximum incidence angle of $72^{\circ}$, the same maximum incidence value was used as an upper bound to define the DAF excitation in the numerical simulations.

\section{Results}

Figures 3(a) and 3(b) show the results obtained for the two considered thicknesses of melamine foam. While the Sabine absorption coefficients obtained with the standard reverberant room method often exceed unity due to the finite size of the sample and to the room effects ${ }^{6,7,21}$ [above $250 \mathrm{~Hz}$ for the 3-in. melamine foam in Fig. 3(a) and above $500 \mathrm{~Hz}$ for the 2-in. melamine foam in Fig. 3(b)], the absorption coefficients calculated with the present approach provide values in good agreement with those obtained from TMM simulations above the $400 \mathrm{~Hz}$ third octave band.

For the 3-in. melamine foam [Fig. 3(a)] and for the $400 \mathrm{~Hz}$ third octave band, the proposed approach gives an absorption coefficient value of 0.63 , while the TMM results and reverberant room provide coefficients of 0.76 and 1.27 , respectively. Above the $400 \mathrm{~Hz}$ third octave band, the difference between absorption coefficients obtained by the present method and TMM results is below 0.1 . For the 2-in. melamine [Fig. 3(b)], and between the 400 and $2000 \mathrm{~Hz}$ third octave bands, the highest 

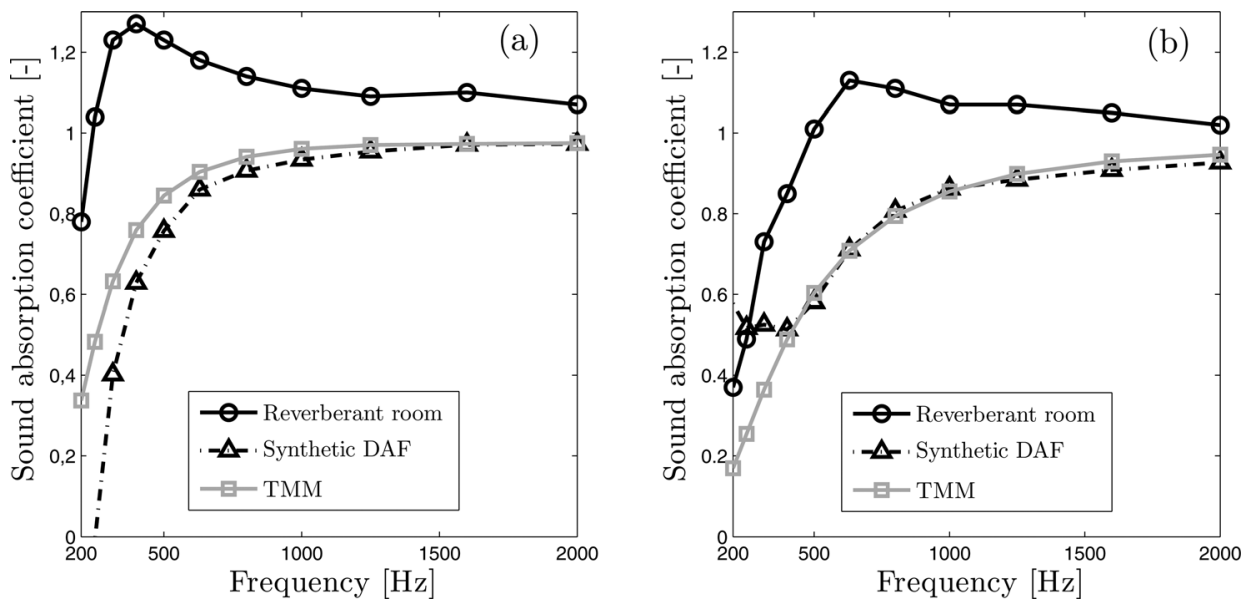

Fig. 3. (a) Absorption coefficient of the 3-inch melamine foam. (b) Absorption coefficient of the 2-in. melamine foam.

deviation between the proposed method and the TMM results occurs at the $1630 \mathrm{~Hz}$ third octave band with a difference of 0.03 between experiments and simulations. A better adequation with TMM results is seen compared to the 3-in. case but was not explained.

Below the $400 \mathrm{~Hz}$ third octave band, the accuracy of the method seems insufficient for both thicknesses with absorption coefficients that either decrease quickly to zero and even negative values for the 3-in. melamine case [Fig. 3(a)] or remain nearly constant for the 2 -in. melamine case [Fig. 3(b)]. This adverse effect is mainly thought to be related to the finite side length of the array. The use of Eq. (2) could involve some discrepancies for small values of $k_{0} r$ as commented in Allard and Champoux. ${ }^{17}$ Source positions and their precise positioning could also be optimized by testing other source distributions and using motorized translation axes. The finite size of the sample may also explain these low frequency limitations and discrepancies with TMM results because this simulation was held for a laterally infinite material.

\section{Conclusions}

(1) The estimated absorption coefficients using a synthesized DAF excitation are in good agreement with those obtained from the TMM above a frequency of $400 \mathrm{~Hz}$, within a physical range (i.e., between 0 and 1), and do not exhibit size effects as seen in reverberant room results even if smaller samples were used. Such measurement results could be directly used for room acoustics computations without any corrections. ${ }^{6,21}$

(2) Instead of using sources positioned on arcs of circle and a linear averaging of source contributions, ${ }^{10,12,13}$ a synthetic plane source array combined with adequately weighted reproduction source complex amplitudes provides the basis for a simplified implementation of absorption measurements under a precise and repeatable DAF excitation with a reduced setup and testing time and no need for specific preparation of specimens. The source can be positioned close to the material, and the presented method might open the possibility to estimate the absorption coefficient under any synthetic incident pressure field even if this letter is limited to the DAF case.

(3) Based on presented results, the accuracy of the method is currently insufficient below a frequency of $400 \mathrm{~Hz}$. This could be explained by the finite size of the sample, and possible improvements and optimization of the virtual source antenna are also currently underway using numerical simulations. 


\section{Acknowledgments}

O.R. wishes to thanks Professor Raymond Panneton for fruitful discussions as well as Jean-Philippe Regnard and Maxime Bolduc, who provided a helpful technical support.

\section{References and links}

${ }^{1}$ ISO 354:2003, Acoustics - measurement of sound absorption in a reverberation room (International Standard Organization, Geneva, Switzerland, 2003).

${ }^{2}$ ASTM C423-09a: Standard Test Method for Sound Absorption and Sound Absorption Coefficients by the Reverberation Room Method (ASTM International, West Conshohocken, PA, 2009).

${ }^{3}$ ISO 10534-2, Acoustics-Determination of sound absorption coefficient and impedance in impedance tubes. Part 2: Transfer-function method (International Standard Organization, Geneva, Switzerland, 1998).

${ }^{4}$ ASTM E1050-12, Standard Test Method for Impedance and Absorption of Acoustical Materials Using a Tube, Two Microphones and a Digital Frequency Analysis System (ASTM International, West Conshohocken, PA, 2012).

${ }^{5}$ T. E. Vigran, L. Kelders, W. Lauriks, P. Leclaire, and T. F. Johansen, "Prediction and measurements of the influence of boundary conditions in a standing wave tube," Acta Acust. united Ac., 83, 419-423 (1997).

${ }^{6} \mathrm{~V}$. L. Chrisler, "Dependence of sound absorption upon the area and distribution of the absorbent material,” J. Res. Natl. Bur. Stand. 13(2), 169-187, Research paper RP700 (1934).

${ }^{7}$ A. Nash, "On the reproducibility of measuring random incidence sound absorption," in Proceedings of Internoise 2012, New York (August 19-22, 2012), pp. 1-12.

${ }^{8}$ R. E. Halliwell, "Inter-laboratory variability of sound absorption measurement," J. Acoust. Soc. Am. 73(3), 880-886 (1983).

${ }^{9}$ A. Duval, J.-F. Rondeau, L. Dejaeger, F. Sgard, and N. Atalla, "Diffuse field absorption coefficient simulation of porous materials in small reverberation rooms: Finite size and diffusivity issues," in Proceedings of 10th French Acoustic Congress, Lyon, France (April 12-16, 2010), pp. 1-8.

${ }^{10} \mathrm{Y}$. Takahashi, T. Otsuru, and R. Tomiku, "In situ measurements of surface impedance and absorption coefficients of porous materials using two microphones and ambient noise," Appl. Acoust. 66, 845-865 (2005).

${ }^{11}$ N. Che Din, T. Otsuru, R. Tomiku, N. Okamoto, and K. Asniawaty, "Reproducibility and applicability of ensemble averaged surface normal impedance of materials using an in-situ technique," Acoust. Austral. 41(3), 207-212 (2013).

${ }^{12}$ T. Otsuru, R. Tomiku, N. Bin Che Din, N. Okamoto, and M. Murakami, "Ensemble averaged surface normal impedance of material using an in-situ technique: Preliminary study using boundary element method," J. Acoust. Soc. Am. 125(6), 3784-3791 (2009).

${ }^{13}$ Z. Kuang, C. Ye, and J. Huang, "A method for measuring diffuse-field sound absorption coefficients of materials using parametric loudspeaker," in Proceedings of Symposium on Ultrasonic Electronics, Tokyo, Japan (December 6-8, 2010), Vol. 31, pp. 331-332.

${ }^{14}$ A. Berry, R. Dia, and O. Robin, "A wave field synthesis approach to reproduction of spatially correlated sound fields,” J. Acoust. Soc. Am. 131(2), 1226-1239 (2012).

${ }^{15}$ O. Robin, A. Berry, and S. Moreau, "Reproduction of random pressure fields based on planar nearfield acoustic holography," J. Acoust. Soc. Am. 133(6), 3885-3899 (2013).

${ }^{16}$ O. Robin, A. Berry, S. Moreau, and S. Campeau, "Experimental reproduction of random pressure fields for vibroacoustic testing of plane panels," in Proceedings of the 19th AIAA/CEAS Aeroacoustics Conference, Berlin, Germany (May 27-29, 2013), Paper No. 2013-2027, pp. 1-23.

${ }^{17}$ J. F. Allard and Y. Champoux, "In-situ two-microphone technique for the measurement of the acoustic surface impedance," Noise Control Eng. J. 32(1), 15-23 (1988).

${ }^{18}$ B. Rafaely, "Spatial-temporal correlation of a diffuse sound field," J. Acoust. Soc. Am. 107, 3254-3258 (2000).

${ }^{19}$ O. Doutres, Y. Salissou, N. Atalla, and R. Panneton, "Evaluation of the acoustic and nonacoustic properties of sound absorbing materials using a three-microphone impedance tube," Appl. Acoust. 71(6), 506-509 (2010).

${ }^{20}$ J.-F. Allard and N. Atalla, Propagation of Sound in Porous Media: Modelling Sound Absorbing Materials, 2nd ed. (Wiley, Chichester, UK, 2009), Chap. 7, pp. 137-165 and Chap. 11, pp. 243-281.

${ }^{21}$ C. H. Jeong, "Converting Sabine absorption coefficients to random incidence absorption coefficients," J. Acoust. Soc. Am. 133(6), 3951-3962 (2013). 